\title{
Structures of ClpC1-NTD with potent anti-TB cyclic peptides Rufomycin and Ecumicin: Implications for the mechanism of action and design of therapeutic agents
}

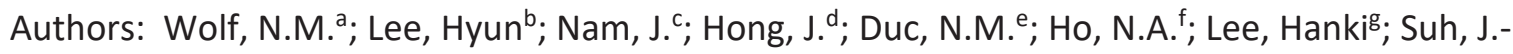
W. ', Pauli, G.F.'; Franzblau, S.G.j; Cho, S. k; Abad-Zapatero, C.

aTR, UIC, 833 S. Wood St., Chicago, IL, 60612, ninawolf@uic.edu

bBiophysics Core at RRC, UIC, 1100 S. Ashland Ave, Chicago, IL 60612, danielhl@uic.edu

'MCP, UIC, 833 S. Wood St., Chicago, IL, 60612, jwan@uic.edu

${ }^{d}$ CNPM, MJU, 116 Myongji-ro, Cheoin-gu, Yongin-si, Gyeonggi-do, 17058 Republic of Korea, jphong@mju.ac.kr eCNPM, MJU, 116 Myongji-ro, Cheoin-gu, Yongin-si, Gyeonggi-do, 17058 Republic of Korea, ducnguyen@mju.ac.kr fCNPM, MJU, 116 Myongji-ro, Cheoin-gu, Yongin-si, Gyeonggi-do, 17058 Republic of Korea, hongocanh@mju.ac.kr gCNPM, MJU, 116 Myongji-ro, Cheoin-gu, Yongin-si, Gyeonggi-do, 17058 Republic of Korea, hklee95@mju.ac.kr ${ }^{h}$ CNPM, MJU, 116 Myongji-ro, Cheoin-gu, Yongin-si, Gyeonggi-do, 17058 Republic of Korea, jwsuh@mju.ac.kr iITR, UIC 833 S. Wood St., Chicago, IL, 60612, gfp@uic.edu IITR, UIC, 833 S. Wood St., Chicago, IL, 60612, sgf@uic.edu kITR, UIC, 833 S. Wood St., Chicago, IL, 60612, jkcno1@uic.edu IITR, UIC, 833 S. Wood St., Chicago, IL, 60612, caz@uic.edu

Abstract: The biological processes related to protein homeostasis have been recently established as crucial pathways for therapeutic intervention. Proteins of particular interest are the $\mathrm{ClpC} 1 / \mathrm{ClpP} 1 / \mathrm{ClpP} 2$ proteosome complex. We present the structures of two potent anti-TB macrocyclic peptides: Rufomycin and Ecumicin complexed with the N-terminal domain of ClpC1. Crystals of ClpC1-NTD-Rufomycin were cubic ( $\mathrm{a}=109.95 \AA$, S. G. P4 $132 ; 1$ complex per a.u.) diffracting to $1.4 \AA$ resolution, and the ones for ClpC1-NTD-Ecumicin were monoclinic ( $a=80.0 \AA$, $b=112.0 \AA$, $c=130.0 \AA$, $\beta=90.07^{\circ}, \mathrm{S}$. G. P2 1 ; 12 complexes per a.u.) diffracting to $2.5 \AA$. Both structures were solved by molecular replacement using the self-rotation function to resolve space group ambiguities. The two structures revealed unique features that could be relevant to understand the mechanism of action of these potent macrocycles. The structure of the macrocyclic depsi-peptide Ecumicin complex showed a unique 1:2 (protein: ligand) stoichiometry exploiting the intra-molecular dyad in the $\alpha$-helical fold of the target $\mathrm{N}$-terminal domain. The structure of the smaller heptapeptide Rufomycin complex unveiled a covalent bond between the SD atom of Met1 and an open epoxide ring, closed in the isolated ligand. The two structures will be compared and the implications for possible different modes of action will be presented. Insights for the design of smaller therapeutic entities against Mycobacterium tuberculosis will be discussed.

Acknowledgment

This work was supported by the Cooperative Research Program for Agriculture Science \& Technology Development (Project no. PJ01328403), Rural Development Administration, Republic of Korea. 